\title{
Voto latino y su influencia electoral en Estados Unidos
} The Latino Vote and Its Influence in U.S. Elections

\author{
DANiel TACHer CONTRERAs*
}

\section{RESUMEN}

El presente artículo analiza la importancia del voto latino en las elecciones presidenciales de 2020 en Estados Unidos, considerando el incremento de esta población en los últimos veinte años y su composición por grupos nacionales. Se documenta dicho crecimiento como producto de los flujos migratorios, en contraste con su tendencia a convertirse en ciudadanos estadounidenses, que afecta su peso electoral. Por último, se establece su ubicación geográfica a fin de considerar el peso electoral en los "estados columpio" (swing states), donde tiene lugar la mayor competitividad para la conformación del Colegio Electoral.

Palabras clave: voto latino, elecciones en Estados Unidos, migrantes.

\section{ABSTRACT}

This article analyzes the importance of the Latino vote in the 2020 U.S. presidential elections, taking into account the growth of this sector of the population in the last 20 years and its composition by national groups of origin. The author documents this sector's demographic growth as a result of immigration, contrasted with its tendency to become U.S. citizens, thus affecting its electoral weight. Finally, he describes Latinos' geographical location to consider this community's electoral weight in "battleground states" or "swing states," where there is more competition for winning seats in the Electoral College.

Key words: Latino vote, U.S. elections, migrants.

\footnotetext{
*Universidad Autónoma de la Ciudad de México (UACM), plantel Casa Libertad, <dtacher@gmail.com>.
} 


\section{INTRODUCCIÓN}

La elección presidencial en Estados Unidos se caracteriza por ser un proceso indirecto, cuyo punto principal es la elección de un Colegio Electoral, conformado por quinientos treinta y ocho miembros, mediante mayoría simple, a partir de listas cerradas y bloqueadas de circunscripciones plurinominales basadas en su división política. Los miembros de este Colegio son propuestos por los partidos a través de dichas listas que el elector no puede modificar; a pesar de ser circunscripciones plurinominales, los escaños no se reparten proporcionalmente. La fórmula electoral de mayoría simple se traduce en que el ganador obtiene todo en cada estado, con excepción de Nebraska y Maine, en donde los miembros del Colegio Electoral se eligen de forma individual y no a partir de listas estatales.

Estas particularidades del sistema electoral han generado, en cuatro ocasiones, que los triunfadores de los votos del Colegio Electoral no sean quienes obtuvieron la mayoría de los sufragios emitidos por los electores. También determinan que el estudio de los votantes en Estados Unidos se realice en forma casuística, analizando el comportamiento estado por estado. Si bien, se pueden observar en términos generales las características del electorado estadounidense, deben analizarse por separado el peso y la importancia específicos de cada grupo de electores según el género, origen étnico, credo, nivel etario, etcétera.

En el caso del voto latino, es indispensable analizar los cambios demográficos en los últimos veinte años, producto de los intensos flujos migratorios provenientes de América Latina, a los que se debe contrastar con los de otra procedencia y composición étnica con el fin de determinar su relevancia electoral. En primer lugar, es necesario considerar la capacidad para integrarse al país de llegada mediante el proceso de obtención de la ciudadanía. En segundo lugar, la importancia de su ubicación geográfica y la relación con los votos que corresponden a cada estado para la conformación del Colegio Electoral.

En el caso de la comunidad latina, donde los mexicanos son el grupo mayoritario, el estudio de su importancia electoral es atravesado por más factores, pues, a diferencia de otros grupos étnicos, está integrada por personas de diverso origen nacional, lo que obliga a reconocer sus particularidades, las cuales inciden en su grado de integración a Estados Unidos como ciudadanos y en su distribución territorial.

En el presente artículo, se examinan tres factores por considerar y que subrayan la importancia del voto latino en la elección presidencial de 2020. En primer lugar, qué se ha entendido por comunidad latina, en este caso, un conjunto "panétnico" conformado por al menos catorce grupos nacionales. En segundo lugar, su crecimiento demográfico en los últimos años, enfatizando la incorporación de los migrantes. 
Por último, se revisa su ubicación geográfica considerando los estados columpio (swing states), también llamados estados púrpura (purple states) o de batalla (battleground states).

\section{¿QUÉ ES UN LATINO?}

La denominación "hispano" o, en este caso, "latino" comenzó a utilizarse en los años setenta del siglo pasado en el censo de Estados Unidos para identificar a todos los grupos étnicos hispanohablantes (Mora, 2014). El uso ha generado controversia sobre su capacidad de clasificar a un grupo al que se presume con un pasado, cultura o rasgos de identidad comunes. A pesar de que se sostiene que esta homogenización invisibiliza la diversidad que caracteriza a esta comunidad, para Mora (2014) tiene una utilidad política, pues permite impulsar su presencia para acceder a mayores espacios de representación y participación.

A pesar de los debates generados por tales términos, han ganado aceptación (Taylor et al., 2012). Las encuestas han mostrado aprobación a su uso entre la propia comunidad aludida, aunque al referirse a las personas se prefería mencionar su lugar de origen; no obstante, en la bibliografía especializada en el comportamiento del electorado estadounidense, ambos términos han sido utilizados al estudiar las actitudes políticas de este núcleo panétnico.

En la bibliografía sobre el tema, puede observarse que hasta mediados de los años ochenta el estudio del comportamiento político de los latinos -término que aquí utilizaremos- se centraba en las actitudes de la comunidad de origen mexicano, describiendo su actividad organizacional y su proceso de adaptación en Estados Unidos (Gómez-Quiñones, 1990); sin embargo, estas investigaciones fueron refinándose con miras a comprender el comportamiento político de una comunidad más amplia, en este caso, la de los latinos o hispanos, términos, como mencionamos, empleados de manera indistinta. De acuerdo con Jones-Correa y Leal (1996), la formación de una identidad panétnica ha sido secundaria en relación con el origen nacional; así, la descripción del latino (o hispano) se ha consolidado como una categoría social que actúa como clasificación caracterizada por un origen común.

La consolidación de esta identidad ha generado cuestionamientos sobre quiénes pueden ser considerados latinos. El común denominador es el idioma y se ha reforzado con la introducción de esta categoría en los censos. En el año 2000 se permitió a las personas indicar si se identificaban con dos o más grupos étnicos. El 93.5 por ciento de los latinos se ubicó únicamente como tal, lo que, de acuerdo con Landale y Oropesa (2002), muestra su definición como grupo étnico. 
Las categorías de latino o hispano fueron expandiéndose principalmente para, por un lado, identificar y asociar a una comunidad con intereses comunes, que se expresaban en el espacio público de forma colectiva a partir de factores como el uso del español o el bilingüismo, el lugar de origen, los estratos generacionales diferenciados de una comunidad de otra, la movilidad social y los niveles de percepción sobre la discriminación estructural (Jensen et al., 2006; Portes y Mcleod, 1996); por el otro, para identificar a este grupo con un enfoque político estudiando la importancia del contexto y estructuras sociales como variables para entender el comportamiento político (Sanchez, 2006; Stokes, 2003). El núcleo duro de los estudios sobre dicho comportamiento ha utilizado variables sociodemográficas (nivel educativo, ingreso, ocupación, etcétera) para explicar patrones de acción política (Marshall, 2001); sin embargo, de acuerdo con Sanchez (2006), en el caso de los latinos, salvo las variables relacionadas con la educación, en general han sido poco efectivas. En contraste, el origen nacional y la identidad panétnica muestran correlaciones positivas con la participación política, organización, activismo y apoyo político-electoral a candidatos latinos.

En ese contexto, es sustancial comprender que esta comunidad está conformada por ciudadanos estadounidenses de ascendencia latina, migrantes naturalizados, indocumentados e hijos de migrantes, por lo que las familias suelen tener miembros con diferentes estatus migratorios. Este segmento social creció en las primeras dos décadas del siglo xxi un 91.87 por ciento. En números absolutos, según la oficina del Censo, en el año 2000 había 31500000 y se espera que en 2020 llegue a los 60500000.

En materia de participación política, Johnson y sus colegas (2003) destacan la importancia de la comunicación bilingüe y el dominio del inglés como medio de acceso a la información sobre procesos e instituciones políticas en Estados Unidos. En particular, documentan el aumento de los medios de comunicación en español enfocados a esta comunidad como un indicador de su incorporación al debate público.

Otro elemento que influye en su actividad política es la vinculación transnacional, al establecer redes entre sus comunidades de residencia y de origen (Jones-Correa, 1998). Como señala Escobar (2004), las relaciones transnacionales incluyen envío de remesas, movimientos circulares migratorios, asociaciones de clubes de oriundos, vinculación con la política de sus lugares de origen y fortalecimiento de la ciudadanía dual.

De acuerdo con DeSipio (2002), la naturalización es la expresión política más significativa en la comunidad de migrantes latinos. En el caso de los mexicanos, la modificación constitucional de 1996, que garantiza no perder la nacionalidad mexicana al adquirir otra, y los efectos de las movilizaciones contra la Propuesta 187 en California (mediante la cual se pretendía negar servicios sociales a migrantes indocumentados) no sólo impulsaron la naturalización de este sector, sino también movilizaron y politizaron al resto de la comunidad latina (Pantoja et al., 2002). 
Si bien demográficamente los latinos se han convertido en la principal minoría, el incremento de su presencia en los padrones electorales sigue siendo lento. En algunos lugares, el tamaño, su participación electoral o su cohesión política sigue siendo insuficiente para impulsar políticos de ese origen; sin embargo, su participación electoral adquiere importancia entre electorados volubles y en coaliciones; por lo anterior, su impacto en los procesos electorales ha sido un gran tema de interés en los últimos años. Partidos y candidatos han desarrollado estrategias para captar este voto, y el "mundo latino" en Estados Unidos toma un nuevo lugar donde la exclusión no tiene cabida, a pesar de las continuas controversias sobre la política migratoria y los esfuerzos (principalmente impulsados por políticos republicanos, pero no de forma exclusiva) por reducir la participación de esta comunidad.

\section{Agendas políticas}

El incremento del electorado latino se ha sostenido en los últimos treinta años, desde la elección de 1988, en la cual solamente constituían el 3.7 por ciento. Para 2012 eran el 10 por ciento y en 2016, el 11 por ciento. En cuanto a su participación, los datos señalan un promedio por debajo de la mitad de su potencial: en la elección de 2008 votó el 49.9 por ciento; en 2012, el 48 por ciento y en 2016, el 47.6 por ciento.

Como se puede observar, a pesar del crecimiento del electorado latino, su participación sigue sin reflejar su relación. Su presencia crece, pero sigue silenciosamente en la agenda. El reto en cada comicio es conocer la forma en que los candidatos y los partidos lograrán captar a este universo de votantes. A pesar de que suele asociarse el voto latino con la agenda migrante, las últimas elecciones plantearon nuevas interrogantes sobre su comportamiento político e influencia en la elección presidencial de 2020.

De acuerdo con Galbraith y Callister (2020), en la elección presidencial de 2016, Donald Trump logró aumentar el porcentaje de latinos que han votado por algún candidato republicano a la presidencia. A pesar de que su campaña se asoció con sus declaraciones antiinmigrantes, particularmente antimexicanas, el 29 por ciento de los votantes latinos lo apoyó. En su estudio, los autores encontraron que las encuestas sobre la elección de 2016 entre personas de ese sector reflejaron que entre los temas de agenda que les interesan están la economía, la educación y la salud, por encima de la política migratoria, aunque la violencia y discriminación contra minorías constituyen sus principales preocupaciones.

Kumar y Ordoñez (2018) coinciden en señalar que el votante latino ha dejado de considerar un tema prioritario las regulaciones migratorias. Las viejas explicaciones de la filiación partidista con el Partido Demócrata se topan con nuevos cuestionamientos. 
Los hallazgos de estos estudios muestran un grupo más plural cruzado por diferencias sociales, de origen y económicas.

Intentar predecir la orientación del voto latino como si fuera un bloque monolítico significa no ver su capacidad de construir alianzas con otros grupos minoritarios, las representaciones sociales que tienen de sí mismos y de ser "americanos". Las nuevas preguntas sobre el electorado latino deberán mirar las particularidades regionales que implican diferentes problemáticas para esta comunidad.

Durante mucho tiempo, se construyó la imagen de una identidad común para este grupo diverso; sin embargo, esta misma heterogeneidad también refleja agendas diversas que son los nuevos objetivos que enarbolan los candidatos y partidos para acercarse a este electorado. Como todo grupo de electores, calculan sus beneficios futuros en función de las ofertas políticas. En el estudio demográfico de la comunidad latina, puede observarse el perfil del votante actual y futuro de este sector, que será la base de las estrategias políticas de partidos y candidatos para acercarse y obtener sus votos, los cuales seguirán creciendo y tendrán la posibilidad de influir en el resultado.

Como observaron Galbraith y Callister (2020), asociar al votante latino con el inmigrante es un estereotipo que poco ayuda a comprender sus principales preocupaciones. Considerar a dicho elector como uno nacido en el extranjero y culturalmente aislado del resto de la población ha llevado a concluir que su principal tema es la migración. La elección de 2016 mostró que, si bien la retórica antiinmigrante asociada con una actitud antilatino hace recordar los movimientos latinos de los años noventa, en el siglo XXI ese votante se siente más preocupado por temas de la agenda social.

\section{Demografía electoral}

Como se ha señalado, el crecimiento demográfico de la población latina y su presencia cada vez mayor en el padrón electoral han sido materia de estudios recientes. La elección presidencial de 2020 representa un nuevo hito para el análisis del incremento de ese voto y su capacidad de influir en el resultado.

Demográficamente, por primera vez los segmentos étnicos identificados como no blancos conforman un tercio de la población; esta nueva distribución se explica por el crecimiento de los grupos latinos y asiáticos. La población latina, convertida en el segundo grupo étnico, supera por primera ocasión a los afroamericanos, al representar el 18.2 por ciento del total, mientras que los afroamericanos son el 12.7 por ciento y los asiáticos, el 5.6 por ciento (U.S. Census Bureau, 2020).

La población latina mantiene su composición histórica. Si bien son una comunidad diversa integrada por catorce grupos nacionales, los mexicanos, como mencionamos, 
siguen siendo mayoría (el 61.89 por ciento), seguidos de los puertorriqueños (el 9.69 por ciento) y los cubanos, estimados en un 3.95 por ciento.

\section{Gráfica 1}

COMPOSICIÓN DE LA COMUNIDAD LATINA (\%)

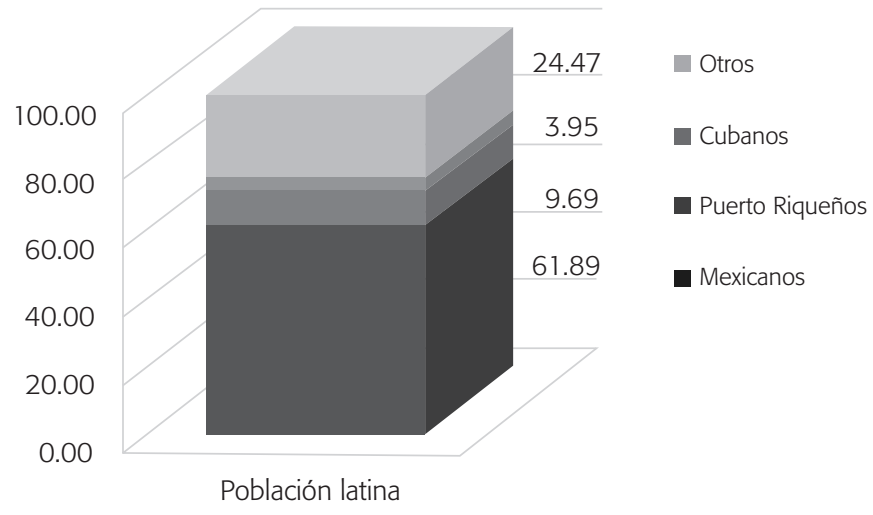

Fuente: Elaboración propia con información de U.S. Census Bureau, 2020.

La importancia demográfica de la población latina calculada en 59743000 habitantes contrasta con su peso electoral. Los miembros de este sector que pueden votar son 29829 000, esto es, el 12.77 por ciento del electorado total en Estados Unidos, que asciende a 233677000 habitantes. Lo anterior se explica porque el 34.80 por ciento nació en el extranjero y sólo el 37.6 por ciento es ciudadano estadounidense.

\section{Gráfica 2}

POBLACIÓN LATINA EN ESTADOS UNIDOS

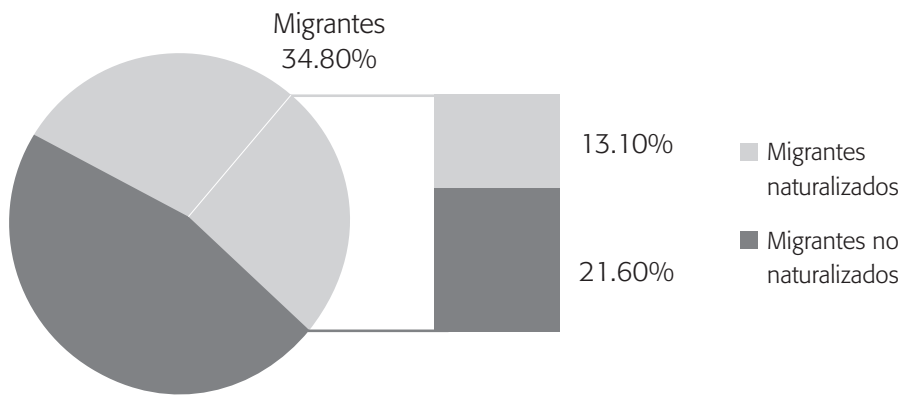

Nacidos en Estados Unidos

$65.20 \%$

Fuente: Elaboración propia con información de U.S. Census Bureau, 2020. 
También se debe considerar la juventud de la comunidad latina. De un total de 73352000 personas menores de dieciocho años en Estados Unidos, los latinos representan el 25 por ciento, lo que los convierte en el segundo grupo en esa categoría. Dentro de la propia comunidad latina, el 32.4 por ciento es menor de dieciocho años. Considerando factores como estatus migratorio y edad, el 49.9 por ciento de los latinos podría ejercer su derecho al voto.

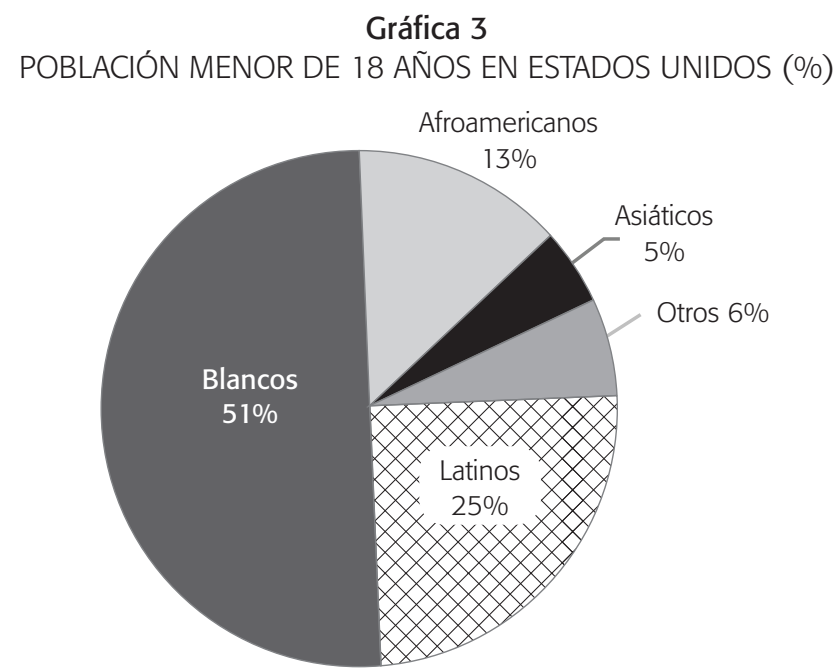

Fuente: Elaboración propia con información de U.S. Census Bureau, 2020.

Los migrantes naturalizados deben cumplir con algunos requisitos de elegibilidad antes de poder votar. Entre los principales, se encuentra demostrar residencia continua en el país, conocimiento (básico) del inglés, así como sobre la historia y gobierno de Estados Unidos. A pesar de lo anterior, el crecimiento de la comunidad en los últimos veinte años supera el de los afroamericanos. En el año 2000, los latinos representaban el 7 por ciento del electorado, mientras que los afroamericanos han mantenido una presencia de alrededor del 12 por ciento. Por otro lado, en la elección de 2020 habrá el mayor número de migrantes naturalizados con posibilidad de votar. En 2000, ese sector estuvo integrado por 12000000 de personas, pero para 2020 se calcula que podría casi duplicarse llegando a los 23200000 (Budiman et al., 2020), mientras que el crecimiento de la población con edad para votar pasó de 181000000 en el año 2000 a 233667000 en 2020.

La población migrante en Estados Unidos (ciudadanos o no) son 45820000 de personas provenientes de prácticamente todos los países. La tendencia del origen se 
ha modificado en los últimos cincuenta años: mientras que en la década de los sesenta del siglo xx provenían principalmente de Europa, en la actualidad la mayoría son originarios de Latinoamérica y Asia. Los migrantes asiáticos crecieron un 122 por ciento en los últimos veinte años, mientras que los latinos lo hicieron un 96 por ciento. Destaca en este crecimiento demográfico la presencia de mexicanos, quienes para 2020 constituyen el 26 por ciento del total.

La relación de migrantes naturalizados por región de origen es otro factor por considerar para entender su peso electoral. En el caso de los migrantes asiáticos, el 57.2 por ciento se ha naturalizado, en contraste con el 40.5 por ciento de los latinoamericanos. Nuevamente los mexicanos resaltan por su bajo índice de naturalización: un 32.7 por ciento.

En un estudio realizado en 2015 por el Pew Research Center (Passel y Cohn, 2015), el 35 por ciento de los mexicanos con residencia legal (poseedores de green cards) tomó como obstáculos para obtener la ciudadanía cuestiones de tipo personal, más que legales. El estudio señala que el 23 por ciento de los entrevistados identificaron como una barrera su falta de dominio del inglés, el 31 por ciento reconoció que tenía poco interés por realizar el proceso y sólo un 13 por ciento señaló los costos administrativos y financieros como los principales obstáculos (Gonzalez-Barrera, 2017).

De nuevo, es importante señalar la relación entre el número de migrantes que pueden votar y su creciente tasa de participación desde el año 2000. Hasta la elección presidencial de 2016, el 54 por ciento de los migrantes naturalizados ejercieron su derecho al voto, en contraste con el 62 por ciento de los electores nacidos en Estados Unidos (Soergel, 2020).

En cuanto a la ubicación geográfica, hay cuatro estados que, por el tamaño de su población, concentran el mayor número de votantes, incluidos migrantes. California, Nueva York, Florida y Texas concentran el 56 por ciento de los migrantes que pueden votar, es decir, 12320000 personas.

En California se concentra el mayor número de votantes y el más alto porcentaje respecto del total. Así, los 5480000 de migrantes que pueden sufragar representan el 21 por ciento del universo de votantes. Nueva York y Florida también comparten esta relación. En el primer caso, los migrantes que pueden votar son 2530000 y representan el 18 por ciento, mientras en Florida residen 2490000 de ellos, es decir, el 16 por ciento.

Como se ha señalado, los migrantes asiáticos tienen gran presencia en este universo. Si bien los nacidos en México son alrededor de 1500 000, los asiáticos procedentes de Filipinas y Vietnam suman 1034000 de electores. De igual forma, en Texas, los migrantes de origen mexicano son el mayor grupo con 700000 electores; los siguen los nacidos en Vietnam e India con 245000 votantes. 
En el caso de Nueva York, se observa una composición distinta de ese electorado, pues los originarios de Cuba y República Dominicana conforman los grupos más numerosos, sumando 870000 electores. Nueva Jersey es un caso aparte, donde el mayor núcleo está integrado por personas originarias de la India, poco más del 10 por ciento del total.

\begin{tabular}{|c|c|c|c|}
\hline \multicolumn{4}{|c|}{$\begin{array}{c}\text { Cuadro } 1 \\
\text { PRINCIPALES ESTADOS CON MIGRANTES NATURALIZADOS CON POSIBILIDAD } \\
\text { DE VOTAR EN LAS ELECCIONES PRESIDENCIALES DE } 2020\end{array}$} \\
\hline \multicolumn{2}{|c|}{$\begin{array}{l}\text { Estados con migrantes por } \\
\text { números absolutos (en millones) }\end{array}$} & \multicolumn{2}{|c|}{$\begin{array}{l}\text { Estados con mayor proporción } \\
\text { de migrantes elegibles para votar (\%) }\end{array}$} \\
\hline California & 5.48 & California & 21 \\
\hline Nueva York & 2.53 & Nueva Jersey & 19 \\
\hline Florida & 2.49 & Nueva York & 18 \\
\hline Texas & 1.82 & Florida & 16 \\
\hline Nueva Jersey & 1.16 & Hawai & 15 \\
\hline Illinois & 0.9 & Nevada & 14 \\
\hline Massachusetts & 0.62 & Massachusetts & 12 \\
\hline Virginia & 0.55 & Maryland & 11 \\
\hline Washington & 0.52 & Rhode Island & 10 \\
\hline Pensilvania & 0.48 & Connecticut & 10 \\
\hline
\end{tabular}

Además de los estados tradicionalmente receptores de migrantes, en Georgia, Minesota y Carolina del Norte se ha triplicado el número de migrantes elegibles para votar en los últimos veinte años. De los estados señalados, Georgia registró el mayor incremento, el 193 por ciento, en contraste con California donde fue del 63 por ciento. 
Como se ha visto, la importancia demográfica de los migrantes latinos está configurando el nuevo rostro de los electores en Estados Unidos. El 53 por ciento del electorado latino es migrante; sin embargo, el tamaño debe considerarse de acuerdo con su ubicación en la definición de los estados de batalla, columpio o pendulares (battleground states o swing states).

\section{LOS BATTLEGROUND STATES Y EL VOTO LATINO}

Como mencionamos, en Estados Unidos la elección del Ejecutivo es indirecta y no está sujeta a la mayoría legislativa. La ciudadanía vota por la conformación de un Colegio Electoral de 538 miembros. Esta composición surge de la base representativa del Congreso, pero no está vinculada con éste. Los 538 electores representan 100 senadores, 436 representantes y 2 electores del Distrito de Columbia, sin representación en el Senado.

Para comprender el aporcionamiento del Colegio Electoral, es necesario considerar la distribución demográfica con base en la cual se integra la Cámara de Representantes, y así como el principio igualitarista de representación estatal de la Cámara de Senadores. El aporcionamiento de los distritos congresionales equivale a 750378 habitantes por cada distrito. Considerando que ningún estado puede quedar sin representantes, aun estando por debajo del promedio, la desviación de aporcionamiento de la Cámara es del + /-11.37 por ciento; sin embargo, al agregar la representación de la Cámara de Senadores, basada en representantes estatales, según un principio igualitario, otorgando dos escaños a cada uno, el aporcionamiento del Colegio Electoral se distorsiona; de esta forma, éste tiene un aporcionamiento promedio de 608113 habitantes por representante, con una desviación del +/-25.42 por ciento.

Esta última desviación se traduce en que California, con sus más de 39000000 de habitantes, cuenta con 55 votos electorales, mientras que Wyoming, con 584 000, tiene sólo tres.

Por otro lado, el sistema electoral de mayoría simple, con circunscripciones plurinominales de lista cerrada y bloqueada, permite que el partido ganador en un estado obtenga la totalidad de los votos electorales. Los únicos sitios donde éstos pueden dividirse son Nebraska (5) y Maine (4). En estos casos, los escaños del Colegio Electoral se eligen en circunscripciones uninominales y con fórmula de mayoría simple.

De esta manera, para ganar la presidencia, se requieren 270 votos del Colegio Electoral, lo que no necesariamente implica ganar la mayoría de los directamente emitidos por los ciudadanos. En la historia de las elecciones en Estados Unidos, sólo en cuatro ocasiones ha habido presidentes que obtuvieron los votos electorales, pero 
no los populares: en 1876, Rutherford Hayes; en 1888, Benjamin Harrison; en 2000, George W. Bush, y en 2016, Donald Trump.

De acuerdo con las reglas de esta elección, existe la posibilidad de que se presente un empate en la distribución de los votos del Colegio Electoral, toda vez que éste se conforma por un número par. En estos casos, de acuerdo con la duodécima enmienda constitucional, la Cámara de Representantes elegirá al presidente entre los tres candidatos más votados y el Senado, al vicepresidente.

El diseño del sistema electoral estadounidense tiene como consecuencia que los candidatos concentren sus esfuerzos en estados donde se pueden presentar variaciones en el resultado, donde tienden a ser volátiles. Para establecer cuáles son los estados de batalla en 2020, se pueden analizar los resultados de los últimos diez comicios presidenciales. Desde 1980, en 43 estados, un partido ha ganado al menos el 70 por ciento de las elecciones y esto los hace muy constantes en sus resultados, por lo que se los puede considerar "bloqueados" (blackout states). En siete más, los resultados se han dividido entre el 40 y el 60 por ciento de las elecciones (Colorado, Michigan, Nueva Hampshire, Nuevo México, Ohio, Pensilvania, Virginia del Oeste). Solamente Nevada ha dividido al 50 por ciento sus resultados. Por otro lado, en la elección de 2016, once estados se definieron por un margen de menos de 5 puntos porcentuales (Arizona, Carolina del Norte, Colorado, Florida, Maine, Michigan, Minesota, Nevada, Nueva Hampshire, Pensilvania y Wisconsin).

También se debe considerar los efectos de la elección de 2016, en la cual Donald Trump logró reducir el número de estados bloqueados por los demócratas desde 1992. Por primera vez, desde hacía seis elecciones, Pensilvania, Míchigan, Wisconsin y uno de los votos electorales de Maine fueron republicanos; sin embargo, los primeros tres y Nueva Hampshire se definieron por menos de un punto porcentual. Por último, las entidades donde la diferencia fue del 5 por ciento son Arizona, Florida, Nevada y Colorado.

De esta forma, se pueden definir catorce estados donde la volatilidad electoral es el factor determinante para ser considerados "de batalla". En conjunto, representan 159 votos electorales en disputa, por lo que en ellos se concentra la decisión final del voto latino y de los migrantes naturalizados. De esos 159 votos, los latinos pueden influir sustantivamente en 103, que corresponden a ocho estados. En aquéllos donde ese sufragio puede ser definitivo, el porcentaje de votantes es superior a las diferencias electorales de 2016. Los estados que concentran el mayor porcentaje de votantes latinos son Nuevo México, Arizona, Florida, Nevada y Colorado, y aquellos donde el porcentaje de votantes latinos es superior al margen de victoria son Pensilvania, Wisconsin y Michigan. 


\begin{tabular}{|c|c|c|c|}
\hline \multicolumn{4}{|c|}{$\begin{array}{c}\text { Cuadro } 2 \\
\text { RELACIÓN DE “ESTADOS BATALLA" CON RESPECTO AL VOTO LATINO }\end{array}$} \\
\hline Estado & $\begin{array}{l}\text { Votos } \\
\text { electorales }\end{array}$ & $\begin{array}{l}\text { Población latina } \\
\text { que puede votar }\end{array}$ & $\begin{array}{l}\text { Porcentaje que } \\
\text { representan los latinos } \\
\text { del total del padrón } \\
\text { electoral }(\%)\end{array}$ \\
\hline Arizona & 11 & 1188000 & 23.60 \\
\hline Carolina del Norte & 15 & 338000 & 4.40 \\
\hline Colorado & 9 & 659000 & 15.90 \\
\hline Florida & 29 & 3143000 & 20.50 \\
\hline Maine & 4 & 16000 & 1.40 \\
\hline Michigan & 16 & 261000 & 3.50 \\
\hline Minesota & 10 & 127000 & 3.10 \\
\hline Nevada & 6 & 407000 & 19.70 \\
\hline Nueva Hampshire & 4 & 31000 & 2.90 \\
\hline Nuevo México & 2 & 645000 & 42.80 \\
\hline Ohio & 18 & 241000 & 2.70 \\
\hline Pensilvania & 20 & 521000 & 5.30 \\
\hline Virginia del Oeste & 5 & 16000 & 1.10 \\
\hline Wisconsin & 10 & 183000 & 4.20 \\
\hline Total & 159 & 7776000 & \\
\hline
\end{tabular}

Considerando la experiencia histórica, en 56 elecciones presidenciales la importancia del voto latino resalta en estados que han definido a los ganadores. Puede decirse que ningún candidato ha ganado la presidencia perdiendo al mismo tiempo Carolina del Norte y Nueva Hampshire. En estos Estados el voto latino representa el 4.4 y el 2.9 por ciento, respectivamente. En los últimos cuarenta años, ningún republicano ha ganado sin Florida; ahí el voto latino representa el 20.5 por ciento del total. En ese mismo periodo, ningún republicano ha ganado perdiendo Nevada y Colorado, donde los latinos constituyen el 19.7 y el 15.9 por ciento, respectivamente, de los padrones locales. Finalmente, en el mismo lapso, ningún demócrata ha triunfado perdiendo en Pensilvania, donde el voto latino es el 5.3 por ciento. 
Como se ha dicho, el crecimiento de la población latina (enriquecida por los flujos migratorios de los últimos veinte años) ha modificado la demografía de Estados Unidos. La presencia de latinos (residentes y migrantes) en varios estados incide en sus comicios locales, donde hay mayor participación en cargos de elección; sin embargo, en cuanto a la presidencial, la importancia de ese voto, reiteramos, está relacionada con su presencia en los estados de batalla o columpio, que definen la conformación del Colegio Electoral.

\section{Conclusiones}

Las elecciones presidenciales de 2020 vuelven a poner el reflector en el voto latino por su incremento en los últimos años, enriquecido por los flujos migratorios. Aunque no todos los migrantes son ciudadanos estadounidenses, y por lo tanto no todos pueden sufragar, el aumento de ese sector de población es relevante. A pesar de que podrá votar menos del 38 por ciento de los esos migrantes, la proporción se ha incrementado desde el año 2000, cuando solamente un cuarto de los latinos podía votar.

Es importante señalar que el impacto del voto de este segmento está vinculado con el aporte demográfico global en Estados Unidos, pero, en particular, con su presencia en estados de batalla, donde se disputará el 30 por ciento de los votos del Colegio Electoral. Adicionalmente, debe considerarse su capacidad para movilizarse y salir a votar en los comicios locales y, en particular, en la conformación del Congreso. Históricamente, los niveles de participación de ciudadanos afroamericanos, migrantes recién naturalizados, jóvenes y personas con baja identidad partidista han sido bajos en comparación con los de sus contrapartes blancos, adultos mayores y con fuerte identidad partidista.

La movilización de los latinos en los estados de batalla puede influir en el resultado por el porcentaje de votantes que representan. En promedio, el voto latino constituye el 10.79 por ciento en estados donde las elecciones han sido cerradas, con votaciones menores al 5 por ciento y donde las entidades han fluctuado de un partido a otro. Su constante crecimiento hará que elección tras elección se busque entender su importancia electoral y la forma en que la agenda política incorpora sus demandas. Sin duda, el comportamiento de la economía, el acceso a la salud y a la educación son los temas más importantes para el electorado latino, por encima del trato a las minorías étnicas e incluso antes que la política migratoria. 


\section{FUENTES}

Budiman, Abby, Luis Noe-Bustamante y Mark Hugo López

2020 "Naturalized Citizens Make Up Record One-in-Ten U.S. Eligible Voters in 2020", Pew Research Center, 26 de febrero, en <https: / www.pewresearch. org/hispanic/2020 / 02 / 26/ naturalized-citizens-make-up-record-one-inten-u-s-eligible-voters-in-2020/>.

DeSIPIO, LOUIS

2002 "Immigrant Organizing, Civic Outcomes: Civic Engagement, Political Activity, National Attachment, and Identity in Latino Immigrant Communities", $1^{\circ}$ de noviembre, uc Irvine, Center for the Study of Democracy, documento 02-08, en <https:/ / escholarship.org/uc/item/4pf4c1tz>.

ESCOBAR, C.

2004 "Dual Citizenship and Political Participation: Migrants in the Interplay of United States and Colombian Politics", Latino Studies, vol. 2, no. 1, pp. 45-69.

Galbraith, Quinn y Adam Callister

2020 "Why Would Hispanics Vote for Trump? Explaining the Controversy of the 2016 Election", Hispanic Journal of Behavioral Sciences, vol. 42, no. 1, pp. 77-94.

GÓMEZ-QUiÑONES, JUAN

1990 Chicano Politics: Reality and Promise, 1940-1990, Albuquerque, University of New Mexico Press.

GonZALez-BArRera, AnA

2017 "Mexican Lawful Immigrants Among the Least Likely to Become U.S. Citizens", Pew Research Center, 29 de junio, en <https:/ / www.pewresearch. org/hispanic/2017/06/29/ mexican-lawful-immigrants-among-least-likelyto-become-u-s-citizens $/>$.

Jensen, Leif, Jeffrey Harris Cohen, Almeida Jacqueline Toribio,

Gordon F. De Jong y LeILA Rodríguez

2006 "Ethnic Identities, Language, Economic Outcomes among Dominicans in a New Destination", Social Science Quarterly, vol. 87, no. 1, pp. 1088-1099. 
Johnson, Martin, Robert M. Stein y Robert WrinkLe

2003 "Language Choice, Residential Stability, and Voting Among Latino Americans", Social Science Quarterly, vol. 84, no. 2, junio, pp. 412-424.

JoNes-CorReA, MichaEL

1998 Between Two Nations: The Political Predicament of Latinos in New York City, Ithaca, Nueva York, Cornell University Press.

Jones-Correa, Michael y David L. Leal

1996 “Becoming 'Hispanic': Secondary Pan-ethnic Identification among Latin American-Origin Populations in the United States", Hispanic Journal of Behavioral Sciences, vol. 18, no. 2, pp. 214-254.

Kumar, Anita y Franco Ordoñez

2018 "Trump Talks Tough on Immigration in Nevada but It Could Backfire", Impact 2020, 25 de septiembre, en <https:/ / www.mcclatchydc.com/news / po litics-government/white-house/article218719245.html>.

Landale, Nancy S. y R. S. Oropesa

2002 "White, Black, or Puerto Rican? Racial Self-identification among Mainland and Island Puerto Ricans", Social Forces, vol. 81, no. 1, pp. 231-254.

Marshall, Melissa J.

2001 "Does the Shoe Fit? Testing Models of Participation for African-American and Latino Involvement in Local Politics", Urban Affairs Revue, vol. 37, no. 2, pp. 227-248.

MorA, G. CRISTINA

2014 Making Hispanics: How Activists, Bureaucrats, and Media Constructed a New American, Chicago, University of Chicago Press.

Noe-Bustamante, Luis, Abby Budiman y Hugo Lopez Mark

2020 Where Latinos Have the Most Eligible Voters in the 2020 Election, Washington, D. C., Pew Research Center, 31 de enero, en <https:/ / www.pewresearch.org / fact-tank / 2020/01 / 31 / where-latinos-have-the-most-eligible-voters-in-the2020-election / > 
Pantoja, Adrian, Ricardo Ramirez y Gary M. Segura

2002 "Citizens by Choice, Voters by Necessity: Patterns in Political Mobilization by Naturalized Latinos", Political Research Quarterly, vol. 54, no. 4, pp. 729-750.

Passel, Jefrerey S. y D’Vera Cohn

2018 "U.S. Unauthorized Immigrant Total Dips to Lowest Level in a Decade", Pew Research Center, 27 de noviembre, en <https: / / www.pewresearch.org / hispanic/wp-content/ uploads / sites / 5/2019/03/Pew-Research-Center_201811-27_U-S-Unauthorized-Immigrants-Total-Dips_Updated-2019-06-25.pdf>.

2015 Immigrant Workers in Production, Construction Jobs Falls Since 2007: In States, Hospitality, Manufacturing and Construction Are Top Industries, Washington, D.C., Pew Research Center, marzo, en <https: / / www.pewresearch.org/hispanic/ 2015/03/26/share-of-unauthorized-immigrant-workers-in-productionconstruction-jobs-falls-since-2007/>.

Portes, Alejandro y Dag MacLeod

1996 "What Shall I Call Myself? Hispanic Identity Formation in the Second Generation", Ethnic and Racial Studies, vol. 19, no. 3, pp. 523-547.

SANCHEZ, GABRIEL R.

2006 "The Role of Group Consciousness in Political Participation Among Latinos in the United States", American Politics Research, vol. 34, no. 4, pp. 427-450.

SOERGEL, ANDREW

2020 "In 2020, 1 in 5 California Voters Will Be Foreign-Born", U.S. News, 28 de febrero, en <https: / / www.usnews.com/news / best-states / articles / 2020-02-28 / on-election-day-2020-1-in-5-california-voters-will-be-foreign-born>.

STOKES, ATIYA KAI

2003 "Latino Group Consciousness and Political Participation", American Politics Research, vol. 31, no. 4, pp. 361-378.

Taylor, Paul, Mark Hugo Lopez, Jessica Martinez y Gabriel Velasco

2012 "When Labels Don't Fit: Hispanics and Their Views of Identity", Pew Research Center, 4 de abril, en <https: / / www.pewhispanic.org/2012/04/04/ when-labels-dont-fit-hispanics-and-their-views-of-identity/>. 


\section{U.S. Census Bureau}

2020 "National Population Totals and Components of Change: 2010-2019", en <https: / / www.census.gov/ data/tables / time-series / demo/popest/2010snational-total.html>.

2019 "2019 National and State Population Estimates", en <https:/ / www.census. gov / newsroom/ press-kits / 2019/national-state-estimates.html>, consultada el 12 de mayo de 2020.

2003 "Census 2000 РнС-т-25. Migration by Origin for the Population 5 Years and Over for the United States, Regions, States, and Puerto Rico: 2000", cuadro 1, 30 de octubre, en <https://www2.census.gov/programs-surveys/decen nial/2000/phc/phc-t-25/tab01.pdf?\#>. 